\title{
Mucormycosis: A black fungus - post covid complication, clinical and pathogenic approaches and isolation and characterization of black fungus with correlate its current perspectives of biomedical waste management
}

\author{
Ajay kumar sahu 1, ${ }^{*}$, BS Arpita 2, Rekita Mohanty 2, Subhashree Dash 3, Purabi Baral 1, Surya Mishra 3, Pinki \\ Samal ${ }^{4}$, Bibekananda Pradhan ${ }^{5}$, Bhagyashree Nanda ${ }^{6}$ and Pragnyasini Rout ${ }^{7}$ \\ ${ }^{1}$ Department of Clinical Microbiology, Sum Ultimate Medicare, Bhubaneswar, Odisha, India. \\ ${ }^{2}$ Centre of Biotechnology, SOA University, Bhubaneswar, Odisha, India. \\ ${ }^{3}$ Department of Biotechnology, Utkal University, Bhubaneswar, Odisha, India. \\ ${ }^{4}$ Department of Biotechnology, Sambalpur University, Odisha, India. \\ ${ }^{5}$ Department of Microbiology, PHEO, Odisha, India. \\ ${ }^{6}$ Department of Bioinformatics, OUAT, Odisha, India. \\ ${ }^{7}$ Department of Biotechnology, MITS School of Biotechnology, Utkal University, Bhubaneswar, Odisha, India.
}

GSC Biological and Pharmaceutical Sciences, 2021, 16(03), 059-070

Publication history: Received on 30 July 2021; revised on 04 September 2021; accepted on 06 September 2021

Article DOI: https://doi.org/10.30574/gscbps.2021.16.3.0258

\begin{abstract}
The clinically relevant black yeasts and their relatives, i.e., members of the Ascomycete order Chaetothyriales. In order to understand the pathology of these fungi it is essential to know their natural ecological niche. From a relatively low degree of molecular variability of the black yeast Exophiala dermatitidis, potential agent of brain infections in patients from East Asia, it is concluded that this species is an emerging pathogen, currently going through a process of active speciation. It is found to be an oligotrophic fungus in hot, moist environments, such as steam baths. CladophialophoraFonsecaea- and Ramichloridium-like strains, known in humans as agents of chromoblastomycosis, are frequently found on rotten plant material, but the fungal molecular diversity in the environment is much higher than that on the human patient, so that it is difficult to trace the etiological agents of the disease with precision, Human to human transmitted disease is the game of corona virus disease (COVID-19) transmission and it had been declared an emergency global pandemic that caused major disastrous in respiratory system to more than five million people and killing more than half a billion deaths across the globe. Besides lower acute respiratory syndrome, there is damage to alveolar with severe inflammatory exudation. COVID-19 patients often have lower immunosuppressive CD4+ T and CD8+ T cells and most patients in intensive care units (ICU) need mechanical ventilation, hence longer stay in hospitals. These patients have been discovered to develop fungal co-infections. COVID-19 patients develop what is known as mucormycosis a black fungal infection which is deadly leading to loss of sight and hearing and eventually death. This chapter will focus on mucormycosis, black fungus caused during post covid complication. This approach has been successful with Cladophialophora carrionii, of which cells resembling muriform cells, the tissue form of chromoblastomycosis, were found to occur in drying spines of cacti. Phagocytosis assays provide a method to distinguish between pathogens and non-pathogens, as the killing rates of strict saprobes proved to be consistently higher than of those species frequently known as agents of disease. therapeutic possibilities for patient chromoblastomycosis. Covid-19 waste is like any other infectious waste and the guidelines for its management are required to be followed in addition to existing practices under regulation. BMWM in context of COVID-19 is a public health concern and is both a legal and social responsibility.
\end{abstract}

Keywords: COVID-19; Mucormycosis; Complications; Isolation and characterization; Black yeasts; Chromoblastomycosis; Phagocytosis

*Corresponding author: Ajay kumar sahu

Department of Clinical Microbiology, Sum Ultimate Medicare, Bhubaneswar, Odisha, India.

Copyright (C) 2021 Author(s) retain the copyright of this article. This article is published under the terms of the Creative Commons Attribution Liscense 4.0. 


\section{Introduction}

Black yeasts have been known since the end of the $19^{\text {th }}$ century, but they still are among the most difficult fungal groups to identify and therefore the knowledge on this group is still only fragmentary. The diagnostic confusion in the past is not surprising, since the taxonomy of black yeasts is now known to be much more complicated than was anticipated. With the application of molecular criteria, a great number of un describe species is encountered. This number is expected to increase even more when detailed studies in biodiversity are performed. Apparently undescribed taxa from the environment and even from human patients are regularly found, and their number is likely to augment exponentially when less commonly Explored sources are sampled.Molecular phylogeny has enabled the attribution of black yeast species to main groups in the fungal kingdom. One of the most interesting. Made in recent years has been the consistent relation of human pathogenic taxa (black yeasts as well as theirs. lamentous counterparts) to a small, clearly delimited group, the order Chaetothyriales, and the family Herpotrichiellaceae in particular. This family is phylogenetically remote from the remaining bitunicate ascomycetes. It has been suggested that the fungi went through a process of rapid diversity cation, probably after having entered a new substratum. It is tempting to speculate that this substratum is the human body.

A fungal pathogen called Aspergillus cause devastating disease in immune compromised hosts. These hosts include haematological malignancies due to fungus being air borne, they cause wreck to lungs guarding frontline defence. Severe viral pulmonary have risen and given birth to pulmonary aspergilliosis (IPA). Most cases are IPA with 7-23\% associated with fatality.This IPA is compared to triple strain corona causing wreckage to lower respiratory tract infections leading to acute respiratory distress syndrome (ARDS). Triple strain corona is considerably high on patients those are in need of ICU furthering them to suffer from mucormycosis. Hence fatality rate is estimated to be high.

It has been understood that black fungus called mucormycosis is complicating the treatment and recovery of COVID-19 patients. There were several reports of patients with invasive aspergillosis and positive growth of Aspergillusfumigatus and Aspergillus-ag in endotracheal aspirate.

While writing this article the total number of cases in India were already around 11,474,605 with more than 3 lakhs active cases. To accommodate the severely ill COVID19 patients, asymptomatic patients were advised for home quarantine and in some cases admitted to temporarily created isolation wards. This massive case number and its consequent healthcare waste is an unprecedented challenge to the existing health services infrastructure.

\section{Epidemiology}

The epidemiology of mucormycosis over the past 2 decades has been complex and, at times, controversial. The exact prevalence and incidence of this infection are unclear because mucormycosis is not reportable. Also, difficulties in diagnosis of mucormycosis in the current era of very low autopsy rates and relative inaccuracy of hospital discharge codes make assessment of the true burden of this infection challenging.

The results of a recent population-based study of mucormycosis in France that relied heavily on hospital discharge codes those in several recent reports from the United States. These studies noted an increasing incidence of mucormycosis, especially in hematology and hematopoietic stem cell transplant (HSCT) units. Although this increased incidence coincided with the introduction of Aspergillus-active agents such as the echinocandins and voriconazole, attributing this trend solely to use of these antifungals may be overly simplistic. Changes in patient profiles and qualitative elements of their immunosuppression may also play equally important roles. In fact, the incidence of this infection was already increasing in the mid-1990s

Other changes in the management of mucormycosis in patients with hematologic cancers at high risk for this infection may increase or decrease the incidence of mucormycosis in this population. Some examples of these changes are worth mentioning. For example, researchers recently found that preexisting iron overload is a predictor of poor outcome and increased adverse effects, such as bacterial and fungal infections (including mucormycosis) and organ toxicity in patients with hematologic malignancies or recipients of HSCTs. Therefore, in view of the availability of the relatively nontoxic oral iron chelator deferasirox, hematologists may be quick to administer this agent early in the natural history of a hematologic cancer. Because deferasirox has activity against Zygomycetes, its use to treat iron overload in patients with hematologic malignancies could theoretically decrease the occurrence of mucormycosis. Others have formulated hypotheses about the indirect consequences of using nonantifungal drugs on the epidemiology of mucormycosis. Specifically, despite the explosion of the diabetes epidemic over the last 2 decades, the incidence of mucormycosis has been shown to be in constant decline in the diabetic population in some, but not all, studies during this time. 
Table 1 Clinical and Research Issues Relating to Mucormycosis Research

\begin{tabular}{|l|}
\hline Clinical and Research Issues Relating to Mucormycosis Research \\
\hline Epidemiology \\
\hline $\begin{array}{l}\text { Prospective international registries to determine the burden of mucormycosis and impact of antifungal use in } \\
\text { diagnosis and identify host groups at risk }\end{array}$ \\
\hline $\begin{array}{l}\text { Development of molecular fingerprinting methods to better define nosocomial versus community-acquired } \\
\text { mucormycosis }\end{array}$ \\
\hline Pathogenesis \\
\hline Comparative studies of the immunopathogenesis of mucormycosis versus aspergillosis \\
\hline Ability to identify innate immune defects in the host that increase risk \\
\hline Innovative animal models in phylogenetically disparate hosts \\
\hline DNA- or antigen-based detection for early diagnosis (eg, before the first radiologic appearance of lesions) \\
\hline Host and radiologic predictors for diagnosis of mucormycosis versus other mold infections \\
\hline
\end{tabular}

\subsection{Pathogenesis}

The pathogenesis of mucormycosis They built these efforts on the results of classic studies from the 1980s documenting the important, unique role of iron acquisition in this pathogenesis. Findings point to iron chelation as an important therapeutic strategy deserving of further exploration. The recent sequencing of the Rhizopus genome and development of molecular tool sets for studying gene function are expected to rapidly increase our understanding of the unique virulence properties of the fungus. Evidence of widespread genome duplication in Rhizopus has shed light on the known attributes of Zygomycetes virulence, specifically, versatility, robust growth, and multidrug resistance. Lists some specific steps to take when studying the molecular pathogenesis of mucormycosis in the postgenomic, we believe that comparative study of the immune pathogenesis of IA and mucormycosis in relevant experimental systems and under different immune suppression scenarios (eg, corticosteroid use versus neutropenia) are equally important.

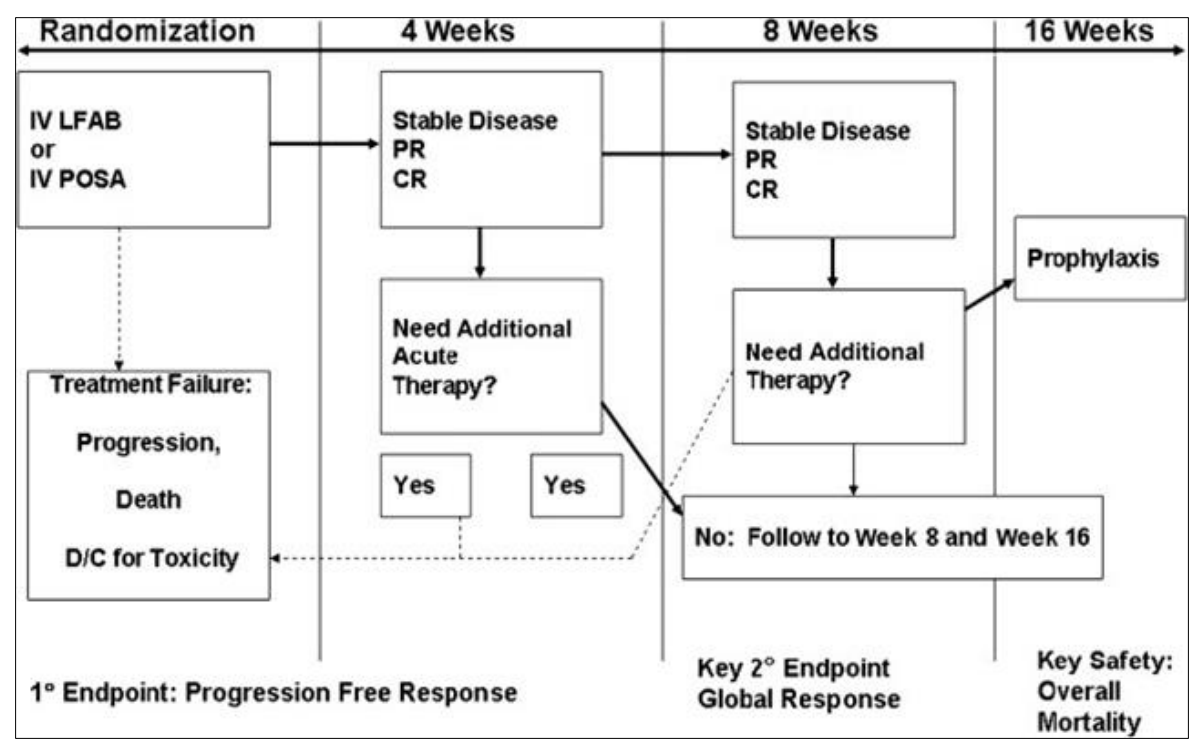

Figure 1 Potential Components of Mucormycosis Risk Stratification at Baseline

Zygomycetes species have great affinity for blood vessels, invade rapidly, and disseminate widely. Furthermore, hemorrhagic necrosis is the hallmark of mucormycotic tissue lesions. However, important questions remain. For example, which endothelial receptors are utilized by Zygomycetes for invasion? Previous data demonstrated the ability of Mucorales to bind, invade, and damage human umbilical vein endothelial cells in vitro. A recent study demonstrated that glucose regulated protein 78 (GRP78) serves as a receptor that promotes the ability of Mucorales to invade endothelial cells lining blood vessels. Elevated concentrations of glucose and iron, consistent with those seen during 
diabetic ketoacidosis, enhance GRP78 expression and resulting invasion and damage of endothelial cells in a receptordependent manner. These findings likely explain the unique susceptibility of diabetic ketoacidosis to mucormycosis.

\section{Infection of neurotropic black yeasts}

The black yeast Exophiala dermatitidis is known from the environment, but also from systemic mycoses in humans. In Southeast Asia fatal cerebral infections are noted in patients which are otherwise in good health. However, the preponderant clinical picture in Europe is subclinical colonization of the lungs of patients with cystic .brosis (CF); the rare systemic cases in this part of the world are mild and occur in immunocompromised patients only. The two clinical pictures are partly caused by members of a single population, as has been determined by random ampli.ed polymorphic DNA (RAPD). The question is whether E. dermatitidis is a contaminant:opportunistic fungus only, as might be concluded from its European occurrence, or whether it should be regarded as a systemic pathogen, as seems apparent from its behaviour in Southeast Asia.To address this question, E. dermatitidis was compared with Pseudallescheria boydii, an environmental species showing neurotropism after temporary coma and aspiration of contaminated water. The taxon displays a remarkable degree of variability in ribosomal DNA (rDNA) internal transcribed spacer (ITS) sequences and polymerase chain reaction (PCR)-. fingerprint data.

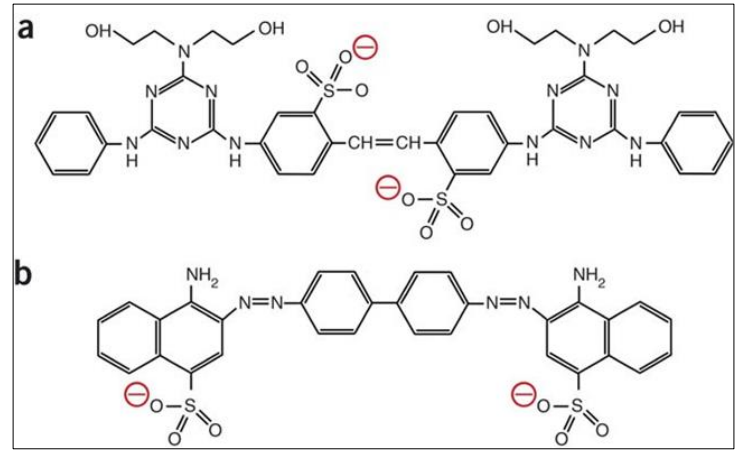

Figure 2 fungal cell wall mutation mechanisms

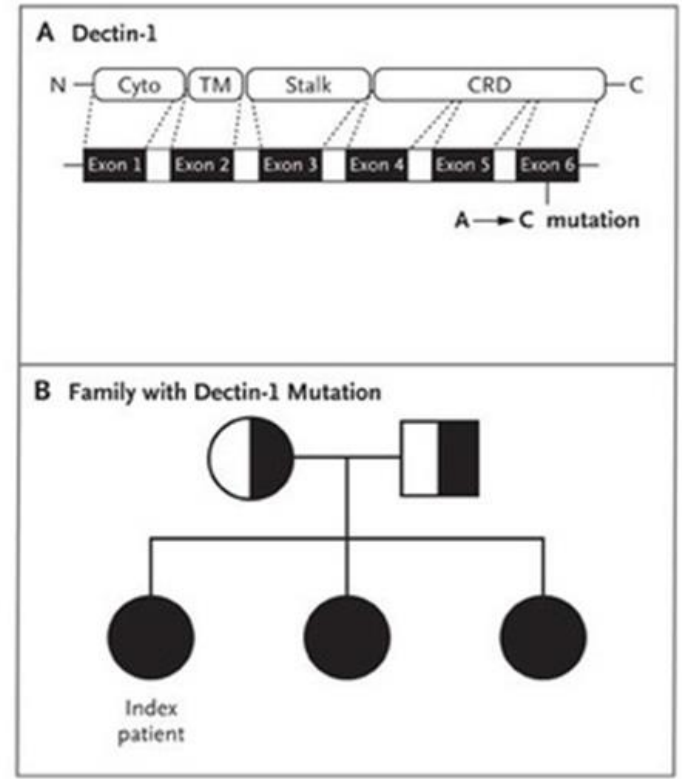

Figure 3 Human Dectin- Deficiency and Mucocutaneous Fungal Infections 
Table 1 Potential Components of Mucormycosis Risk Stratification at Baseline

\begin{tabular}{|l|}
\hline Preenrollment characteristic \\
\hline Age (>40 vs <40 years) \\
\hline Type of hematopoietic stem cell transplant \\
\hline Allogeneic \\
\hline Autologous \\
\hline Relapsed leukemia \\
\hline Interval from diagnosis to surgery \\
\hline Early (1-7 days) \\
\hline Intermediate (8-14 days) \\
\hline Late ( \\
\hline Diabetes control \\
\hline Good days) \\
\hline Poor \\
\hline Staging of mucormycosis \\
\hline Disseminated \\
\hline Rhinocerebral \\
\hline
\end{tabular}

\section{Biomedical waste management rules India}

India implemented Biomedical Waste Management (BMWM) rules first in 1998 and then a more comprehensive legislation- BMWM rules, 2016 and their amendments in 2018 and 2019.COVID19 was declared as a pandemic in March 2020 and India had responded with stringent guidelines from CPCB. CPCBbrought out specific guidelines for handling, treatment and disposal of waste generated during treatment, diagnosis, and quarantine of COVID-19 patients on March 18,2020 , followed by fourth revision on July 17,2020 . These guidelines are required to be followed by all stakeholders in addition to existing practices under BMWM Rules, 2016 as amended.

\subsection{The unique challenge of the novel Corona virus (SARS CoV2)- COVID19}

Knowledge of the virus nature and viability in different environment and on inanimate objects is pertinent for COVID19 waste disposal. COVID19 virus can be transmitted from human to human and also by fomites .SARS CoV19 can remain viable up to $72 \mathrm{~h}$ on plastic and metal surfaces whereas no viable virus can be detected after $24 \mathrm{~h}$ on cardboard and after $4 \mathrm{~h}$ on copper.Human coronavirus can remain infectious on inanimate surfaces for more than 9 days and persistence is reduced at temperature higher than $30{ }^{\circ} \mathrm{C}$.As it has a lipid envelope, it is susceptible to common disinfectants $(0.5 \%$ hypochlorite, $0.5 \%$ hydrogen peroxide, $2 \%$ glutaraldehyde), UV radiation, dry heat and moist heat.COVID19 virus can remain suspended in air for more than $3 \mathrm{~h}$ and can be a source of further infection. As per WHO, airborne transmission of the virus can occur in health care settings where specific medical procedures, called aerosol generating procedures, generate very small droplets $(\leq 5 \mu \mathrm{m})$. Some outbreak reports related to indoor crowded spaces have suggested the possibility of aerosol transmission, combined with droplet transmission, for example, during choir practice, in restaurants or in fitness classes.

\subsection{Challenges for bio medical waste management during COVID19}

Every day of the COVID19 pandemic brings out a new challenge that needs troubleshooting.

- Waste generated during home quarantine period are also potentially infectious. Their safe disposal needs to be addressed.

- Waste generated in newly formed COVID-19 isolation wards, COVID intensive care unit ICU, quarantine homes and COVID care centres need proper management. 
- There is a need of safe burial or cremation of the dead COVID19 patients as this is a matter of safety and sentiment.

- Shedding of the virus in stool, can lead to drinking water contamination if sewage lines fail to operate properly.Sewage overflowing in heavy rainfall and sewage flooding can cause human exposure from aerosolization from sewage water. There is also concern on long term effect on COVID 19 viral shedding in faecal matter in patients as it has environmental implications too.

- $\quad$ During COVID19 pandemic, increased use of biocidal agents, detergents, liquid soaps or simple water used in washing of contaminated material possess a threat to water bodies.

- Environmental problems that may arise from the quantity of COVID-19 waste also need to be addressed.

- PPE is now utilised by general population, patient's attendants visiting hospitals, care centres, office staff in public and private offices, airport staffs and railway officials. These new consumers are either unaware or untrained on the potential hazard of improperly discarded protective gears and etiquettes of PPE.

- COVID 19 testing labs, Vaccine research labs might be handling a huge viral load and any mistake can be catastrophic.

\subsection{WHO guidance on infection prevention and control and waste management}

WHO WASH (Water, Sanitation and Health) has released guidelines for safe handling and management of COVID-19 waste on March 3, 2020 followed by revision on March 24, 2020 and April 23, 2020. The most recent guidelines published on June 29, 2020 include detail of infection control and prevention strategy for COVID-19 patient care and waste management. Any BMW generated must be collected in clearly labelled containers and sharp safe boxes. Adequate manpower professionally trained should be assigned for COVID-19 waste management. Onsite treatment should be preferred as much as possible to reduce mishap. When offsite waste treatment is unavoidable care should be taken for its proper disposal. Use of heavy-duty boots, splash proof aprons, goggles, masks and face shields and adherence to hand hygiene after handling waste is recommended for waste handlers. For the cleaning of environment, non-porous, surfaces effectively, at the outset clean the surfaces with water and detergent followed by a disinfectant for COVID-19 $(0.1 \%$ sodium hypochlorite or $70-90 \%$ ethanol). Contact time of a minimum of 1 min is recommended for ethanol, chlorine-based products and hydrogen peroxide $\geq 0.5 \%$. Thereafter disinfectant residue is rinsed off with clean water. Medical devices and equipment, laundry, food service utensils and medical waste should be managed in accordance with safe routine procedures.

\subsection{CPCB guidelines, 2020}

Table 2 Classification of hazardous medical waste

\begin{tabular}{|c|l|l|}
\hline Sr number & \multicolumn{1}{|c|}{ Sharps } & \multicolumn{1}{|c|}{ Waste entailing risk of injury } \\
\hline 1 & Waste entailing risk of contamination & $\begin{array}{l}\text { Waste containing blood, secretion or } \\
\text { excreta entailing a risk of } \\
\text { contamination }\end{array}$ \\
\hline 2 & Anatomical waste & $\begin{array}{l}\text { Body parts, tissue entailing a risk of } \\
\text { contamination }\end{array}$ \\
\hline 3 & Infection waste & $\begin{array}{l}\text { Waste containing large quantities of } \\
\text { material, substance or culture } \\
\text { entailing the risk of propagating } \\
\text { infection agents }\end{array}$ \\
\hline 5 & Pharmaceutical waste & $\begin{array}{l}\text { Spilled unsed medicine expired drugs } \\
\text { and used medication receptacles }\end{array}$ \\
\hline 6 & Waste containing heavy metal & $\begin{array}{l}\text { Batteries, mercury waste and light } \\
\text { tubes }\end{array}$ \\
\hline & Chemical waste & $\begin{array}{l}\text { Chemical waste, laboratory solvent } \\
\text { disinfectant developer and fixer }\end{array}$ \\
\hline
\end{tabular}

These guidelines for are required to be followed by stakeholders in addition to existing practices under BMW Management Rules, 2016 as amended. These include colour categories, lab waste disposal, PPE disposal, home care waste disposal, solid waste disposal, liquid waste disposal, duties of stakeholders to troubleshoot the problems faced by health care workers and biomedical waste handlers. The fourth revision of guidelines, July 2020 were issued to 
provide revised guidance on segregation of general solid waste and biomedical waste from quarantine centres/homecare/healthcare facilities treating COVID-19 patients and to recommend on disposal of PPEs.

- Waste generated in COVID-19 isolation centres: To reduce the COVID19 contaminated waste it is advised to discard BMW contaminated with COVID-19 patients body fluids and secretions into COVID-19 waste labelled foot operated colour coded bins.General solid waste should be collected separately as per SWM Rules, 2016 .The wet (compostable bags) and dry solid waste bags need to be tied securely in leak-proof bags, sprayed with hypochlorite solution and hand over to authorized waste collector of ULBs on daily basis, without opening them. Masks, tissues and toiletries used by COVID-19 patient can be contaminated with respiratory secretion, hence they need to be discarded into yellow colour bags. COVID19 waste management challenges faced by isolation/ quarantine centres:Training in BMWM in COVID-19 context has to be practiced with social distancing norms (small groups in halls or online training, or onsite training in small groups). All HCWs, individuals, groups working in hospital like doctors, nurses, technicians, sanitation workers and staff who use PPE, should be included. BMWM needs to be practiced with social distancing norms, hand hygiene, PPE, respiratory etiquettes. CPCB mobile application (App.) mandates the COVID-19 BMW colour category wise to be submitted daily to state pollution control board (SPCB). The various categories of BMW as mentioned in BMWM rules 2016 and CPCB guidelines, 2020 as amended is depicted.

- Waste generated during Home Quarantine or Home-Care facilities: Covid-19 patients clinically assigned to as very mild/presymptomatic/asymptomatic can be home isolated. As mentioned in SW rules 2016, general waste classified into bio-degradable, non-biodegradable and domestic hazardous waste. Biodegradable waste and non-bio-degradable waste should be discarded into designated colour coded bags and collected by municipal solid waste collector of municipality or ULBs. General solid waste should comprise of general waste used by suspected quarantined persons and COVID-19 patient at homecare or home quarantine. Only the used masks, gloves and tissues or swabs contaminated with blood/body fluids of COVID-19 patients, including used syringes, medicines, etc., if any generated should be treated as biomedical waste. Masks worn by COVID-19 patients should be disposed in yellow biohazard bag to picked by dedicated waste handler from ULB [4]. In case the ULB has not started such facilities then the mask can be disinfected by 1\% hypochlorite before discarding. Furthermore, it is advised to store used gloves and mask from COVID 19 negative households in paper bags for a minimum of $72 \mathrm{~h}$ and disposing them as general non-bio-degradable waste after mutilation.

- Sewage treatment facilities: Uptill now no proven case of COVID19 was linked to wastewater or sewage aerosol has been reported. Previously many studies had reported that aerosol generated in sewer lines can act as a potential source of coronavirus infection.9,10 Health care facilities with effluent treatment plants need to follow all guidelines for disinfection prior to discharge into general sewage lines. Persons working in effluent treatment plants and wastewater treatment facilities should be equipped with PPE, gloves, masks and goggles.Training the workers on standard precaution, respiratory etiquette, hand hygiene, etc can reduce the risk of any accidental exposure. The reuse of waste water should be avoided during pandemic.

- Waste handlers, waste handling facilities: In house COVID-19 waste transport should be done in closed trolleys with easy manoeuvrable wheels and labelled with COVID-19 waste. These trolleys should be disinfected regularly. All biomedical waste bags should be double-layered, non-chlorinated, leakproof, labelled COVID-19 waste and barcoded and then handed over to CBWTF. All areas involved in COVID19 case management should be provided and serviced only with COVID19 WASTE labelled bins, bags and containers. Waste handlers should be equipped with PPE as mentioned earlier. All waste handlers to be trained with infection prevention measures such as hand hygiene, respiratory etiquettes, social distancing, use of appropriate PPE, etc. via videos and demonstration in local language at regular interval. Designated nodal officer for BMWM of the facility should carry out these trainings

\subsection{Diagnosis}

Early diagnosis of mucormycosis remains challenging and is a major unmet need, causing a bottleneck in devising innovative, effective clinical trials. This is important because delayed treatment clearly impacts the outcome of mucormycosis . Major problems in the diagnosis of mucormycosis include its elusive clinical presentation and frequent occult dissemination, a lack of sensitive nonculture-based diagnostic methods (eg, antigen and molecular detection platforms), and the fact that culture of samples obtained from nonsterile sites (eg, sputum) is neither sensitive nor specific. Tissue-based diagnosis remains the gold standard, although patients frequently are not candidates for biopsy analysis because of thrombocytopenia or hemodynamic instability. Even with tissue-based diagnostic methods, sampling errors and occasional difficulties in differentiating Zygomycetes from distorted hyalohyphomycetes in tissue samples result in false-negative or false-positive results. Furthermore, paradoxically, two-thirds of histopathologically proven mucormycosis cases are culture negative. It has not yet been determined whether optimal processing of infected tissue samples and adaptation of culture conditions to simulate semi-anaerobic growth require further validation. 
Morphologic identification of Zygomycetes at the species level is reasonably accurate, especially when performed in high-volume or reference clinical microbiology laboratories. Of note, carbon-assimilation profiles have been reported to be a promising tool for the precise identification of Zygomycetes. Polymerase chain reaction-based detection of Zygomycetes fungi remains investigational, although recent data reveal that ITS sequencing was promising for identification of Zygomycetes genus/species from culture, frozen sections, or paraffin-embedded sections of infected tissues. Whether detection of volatile compounds produced by Zygomycetes fermentation in patients with mucormycosis is of help in diagnosis is an unexplored area. An equally important unexplored area is identification of diagnostic factors that favor mucormycosis over the more common IA on clinical grounds. Retrospective singleinstitution studies focusing on patients with leukemia and/or recipients of HSCTs identified prior voriconazole exposure, community-acquired pansinusitis, hyperglycemia (eg, steroid-induced), multiple (>10) nodules detected on chest computed tomography scans, pleural effusion, and the reverse halo sign as markers potentially favoring a diagnosis of mucormycosis over a diagnosis of aspergillosis. Prospective validation and similar studies of other populations at risk for mucormycosis are needed. Development of a "scorecard" for diagnosis of mucormycosis would be ideal.

\subsection{Treatment}

The literature on mucormycosis is modestly helpful to clinicians managing this infection. However, "primary" prospective mucormycosis treatment data have yet to be reported. Both salvage and single-institution retrospective studies should be viewed with caution in light of the multiple inherent biases. Multiple controversies remain. For example, host heterogeneity, pleiotropic clinical presentation (at least 6 clinical syndromes), the multitude of Zygomycetes species, factors related to surgery (eg, timing, radicalness), and the influence or correction of underlying metabolic (eg, ketoacidosis) and immunosuppressive (eg, neutrophil recovery in neutropenic patients, steroid tapering in patients receiving corticosteroids) conditions lead to complex and at times highly individualized scenarios for management of mucormycosis. For example, recent studies at The University of Texas MD Anderson Cancer Center showed that a lack of corticosteroid tapering, monocytopenia and persistent neutropenia, and a lack of early treatment of mucormycosis were powerful independent predictors of poor outcome of this infection.

In our view, a successful clinical trial of treatment of mucormycosis hinges on the following factors:

- Enthusiastic participation of all investigators so that as many patients as possible are enrolled and protocol violations are kept to a minimum.

- Risk stratification using a scoring system.depicts a possible scoring system that is based on preenrollment patient characteristics. Unfortunately, such scoring systems have yet to be developed and validated not only for mucormycosis but also for other clinically important fungal infections.

Waste generated during Home Quarantine or Home-Care facilities: Covid-19 patients clinically assigned to as very mild/pre-symptomatic/asymptomatic can be home isolated As mentioned in SW rules 2016, general waste classified into bio-degradable, non-biodegradable and domestic hazardous waste, Biodegradable waste and non-bio-degradable waste should be discarded into designated colour coded bags and collected by municipal solid waste collector of municipality or ULBs. General solid waste should comprise of general waste used by suspected quarantined persons and COVID-19 patient at homecare or home quarantine. Only the used masks, gloves and tissues or swabs contaminated with blood/body fluids of COVID-19 patients, including used syringes, medicines, etc., if any generated should be treated as biomedical waste. Masks worn by COVID-19 patients should be disposed in yellow biohazard bag to picked by dedicated waste handler from ULB. In case the ULB has not started such facilities then the mask can be disinfected by $1 \%$ hypochlorite before discarding. Furthermore, it is advised to store used gloves and mask from COVID 19 negative households in paper bags for a minimum of $72 \mathrm{~h}$ and disposing them as general non-bio-degradable waste after mutilation

Sewage treatment facilities:Uptill now no proven case of COVID19 was linked to wastewater or sewage aerosol has been reported. Previously many studies had reported that aerosol generated in sewer lines can act as a potential source of coronavirus infection.Health care facilities with effluent treatment plants need to follow all guidelines for disinfection prior to discharge into general sewage lines. Persons working in effluent treatment plants and wastewater treatment facilities should be equipped with PPE, gloves, masks and goggles.Training the workers on standard precaution, respiratory etiquette, hand hygiene, etc can reduce the risk of any accidental exposure. The reuse of waste water should be avoided during pandemic.

Safe disposal of personal protective equipment: To reduce volume of PPE and to prevent scarcity,it's reuse has been advised with proper precaution . N95 masks can be reused for 3-4 times by the same person if stored in paper bags and 
kept out of reach of other people, Visors and goggles are made of hard plastic hence can be disinfected by immersing in $1 \%$ sodium hypochlorite or $70 \%$ alcohol for $10 \mathrm{~min}$ Gloves, surgical masks should not be reused and should be discarded in proper colour coded bags after mutilation to prevent pilferage. Masks, shoe cover and head cover made of mixed material with 30-40\% polyethylene plastic fibre should be discarded into yellow bags/bins for incineration. But latex gloves, full plastic PPE, splash-proof apron, plastic coverall, Hazmat suites, visors (face shields) and nitrile gloves need to be discarded into red bags for sterilisation by chemical method, microwaving or autoclaving before being recycled as plastic encapsulated bricks or plastic for road making. At Material Recovery Facilities (MRFs), discarded PPEs containing plastic should be shredded and sent to SPCB authorized plastic waste recyclers, or may be converted into refuse derived fuel (RDF) for co-processing or energy recovery (Waste to Energy Plants or for road making). When these facilities are unavailable the end product from treated PPE, gloves and other plastic materials can be sent to sanitary landfill sites.

\section{Material and methods}

\subsection{Sample collection}

Sample were collected from only the patient who suffer in black fungus disease, as this disease has a new strain its very risky that handle improperness.

Sample collected only of infected person nasal swab ear swab and eye swab or other skin flora.

\subsection{Sample collection procedure}

As black fungus is very deadly sample were collected after were of properly wear of PPE kiit and proper safety assurance.

\subsection{Instruments need for collection}

For collection of black fungus sample sterile swab and sharp blade needs for scrubbing sample and it's should be kept for proper preservation

\subsection{Inoculation procedure}

For inoculation of black fungus sample, we need two pair of media plates as one blood agar and another is SDA media which is helps to grown fungus in adequate number.

\subsection{Incubation}

After inoculation of sample one pair were kept in BOD chamber and another is $37^{\circ} \mathrm{c}$ incubator.

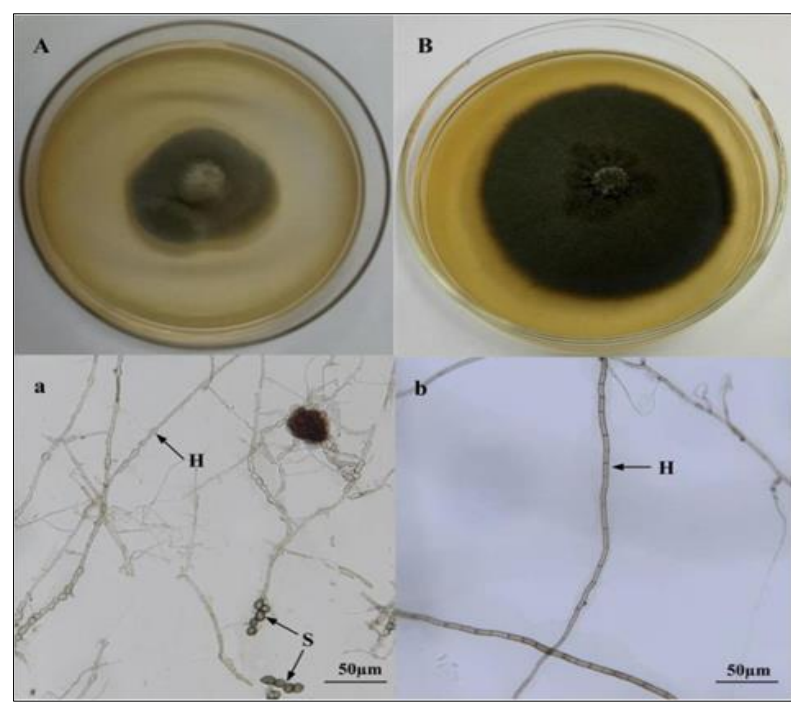

Figure 4 Isolation of black fungus 


\subsection{Screening method}

For screening of black fungus using fungal stain

- Take a clean glass slide.

- Add a drop of Lacto phenol Cotton Blue reagent on a clean and dry slide.

- Sterilize the needle and cool it.

- Then transfer a mycellial mat on fluid and press it gently so that it easily mix with the stain.

- Carefully tease the fungal culture into a thin preparation.

- $\quad$ Place a coverslip on the preparation (mycellial mat). Wait for about 5 minutes.

- Take a blotting paper (tissue paper) and wipe the excess stain.

- Observe first under microscope with low power for screening in low intensity

\subsection{Principle and Interpretation}

- Lactophenol Cotton Blue reagent is used for staining as well as for wet mounting of fungi.

- Lactic acid preserves the fungal structure and clears the tissue.

- Phenol acts as a disinfectant or fungicidal (kill any live organisms).

- Cotton blue it stains the chitin in the fungal cell walls (fungal elements)

\subsection{Microscopic Examination}

Fungal Spores and hyphae are observed under microscope using high power (40X) objective lens after staining with Lactophenol cotton blue.

\section{Results}

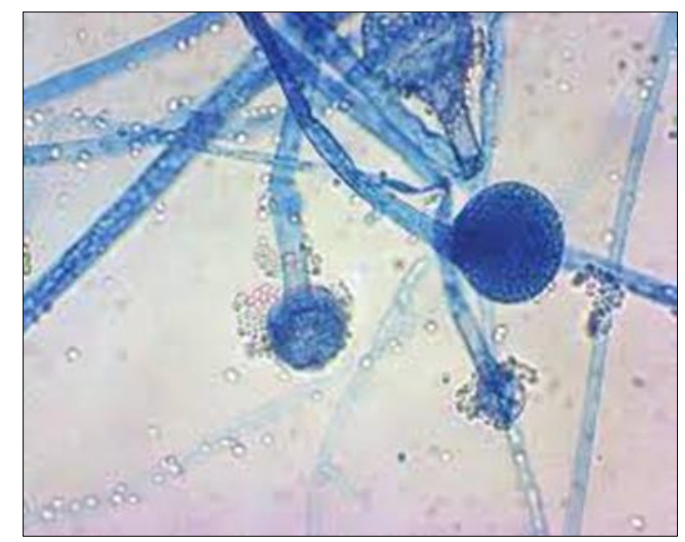

Figure 5 Black fungus screening

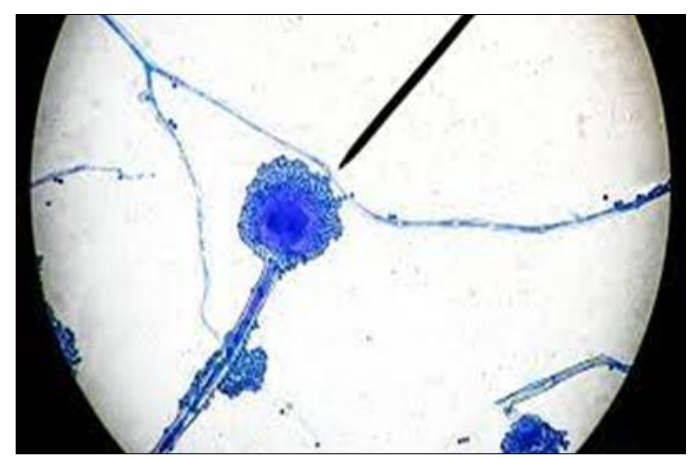

Figure 6 fungal hyphae microscopy view 


\section{Conclusion}

Recent revisions and review in national and international guidelines were specific for COVID19 waste management include the new isolation facilities, laboratories, camps, and care homes. Stringent compliance to the established rules, guidelines and identification of newer support areas are warranted, that can help in reducing the burden and in preventing potential outbreak. Just as for COVID19 management "Identify Isolate and Treat" the same goes for COVID19 waste identify the generator, isolate i.e., manage separately in covid19 waste designated bins, trolleys, and finally treat the waste adequately. The essential principle of BMWM rules in COVID-19 context remain essentially the same based on WHO core principles and with focus on non-burn technologies for BMWM using the best available practices and best available techniques. COVID 19 waste is an important public health, therefore it is a legal and a social responsibility of all stakeholders. Furthermore, without active stakeholders including citizens participation and cooperation, intermixing of biomedical waste with solid waste can endanger the occupational safety of HCWs, society and environment.

Devastating infection had a palpable sense of working in a frontier of clinical mycology. Much work remains ahead of us. By mobilizing resources, organizing, expanding intellectual energy, and thinking innovatively, we can make strides in improving outcomes of mucormycosis.

\section{Compliance with ethical standards}

\section{Acknowledgments}

We thank our patients for providing the inspiration for doing research related to this disease. The author thanks to support staff Hod of Microbiology Dr. Purabi Baral from clinical microbiology department of sum ultimate Medicare and also thanks to team member of utkal university biotechnology department.

\section{Disclosure of conflict of interest}

All the collected data is not copy from any other sources all method was carried out in accordance with approval guideline.

\section{Statement of informed consent}

Informed consent was obtained from all individual participants included in the study.

\section{References}

[1] WHO Coronavirus disease (COVID-19) weekly epidemiological update and weekly operational update. 2020.

[2] Compliance Status Report by CPCB in OA 72 of 2020 (In Re Scientific Disposal of Bio-Medical Waste Arising Out of COVID-19 Treatment- Compliance of BMW Rules. 2016.

[3] MOEF FF. BMWM Rules Gazette of India, extraordinary, Part II. Section. 2016; 3(i): 1-37.

[4] MOEF FF. Central Pollution Control Board. (Revision 4) Guidelines for Handling, Treatment and Disposal of Waste Generated during Treatment/Diagnosis/ Quarantine of COVID-19 Patients.17 July 2020.

[5] WHO FF. Transmission of SARS-CoV-2: Implications for Infection Prevention Precautions. 2020.

[6] Van Doremalen N, Morris DH, Holbrook MG, Gamble A, Williamson BN. Aerosol and surface stability of SARS-CoV2 as compared with SARS-CoV-1. N. Engl. J. Med. 2020; 382(16): P1564-P1567.

[7] Kampf G, Todt D, Pfaender S, Steinmann E. Persistence of coronaviruses on inanimate surfaces and their inactivation with biocidal agents. J. Hosp. Infect. 2020; 104(3): 246-251.

[8] Yaacoub S, Schünemann HJ, Khabsa J. Safe management of bodies of deceased persons with suspected or confirmed COVID-19: a rapid systematic review. BMJ Global Health. 2020; 5.

[9] Tang A, Tong ZD, Wang HL, Dai YX, Li KF, Liu JN, Wen-jie Wu W, Yuan C, Yu M, Li P. Yan JB. Detection of novel coronavirus by RT-PCR in stool specimen from asymptomatic child, China. Emerg. Infect. Dis. 2020; 26(6): P1341-P1343. 
[10] Lodder W, de Roda Husman AM. SARS-CoV-2 in wastewater: potential health risk, but also data source. The lancet. Gastroenterol. Hepatol. 2020; 5(6): P533-P534.

[11] Quilliam RS, Weidmann M, Moresco V, Purshouse H, O'Hara Z, OliverCOVID-19 DM. The environmental implications of shedding SARS-CoV-2 in human faeces. Environ. Int. 2020; 140: 105790.

[12] Nghiem Long, Morgan Branwen, Donner Erica, Short Michael. The COVID-19 Pandemic: Considerations for the Waste and Wastewater Services Sector. Case Studies in Chemical and Environmental Engineering. 2020.

[13] Saadat S, Rawtani D, Hussain CM. 2020; 728.

[14] NGT National Green Tribunal Order regarding COVID-19 waste management dated 24/04/2020.

[15] MOEF CC. Solid Waste Management Rules . 2016. The Gazette of India: Extraordinary. Part II, 3(ii): 8-12.

[16] WHO Laboratory biosafety guidance related to the novel coronavirus (2019-nCoV) Interim guidance.

[17] WHO. Infection Prevention and Control during Health Care when Coronavirus Disease (COVID-19) Is Suspected or Confirmed. 2020.

[18] WHO Water, sanitation, hygiene, and waste management (WASH) for the COVID-19 virus: interim guidance.2020.

[19] Government of India, Ministry of Health \& Family Welfare Revised guidelines for Home Isolation of very mild/pre-symptomatic/asymptomatic COVID-19 cases. 2020.

[20] MOHFW Guidelines on use of masks by public.2020.

[21] MOHFW India Advisory on re-processing and re-use of eye-protection - Goggles.2020.

[22] The National Institute for Occupational Safety and Health Niosh Recommended guidance for extended use and limited reuse of $\mathrm{N} 95$ filtering facepiece respirators in healthcare settings.2020.

[23] Capoor MR, Bhowmik KT. Current perspectives on biomedical waste management: rules, conventions and treatment technologies. Indian J. Med. Microbiol. 2017; 35: 157-164.

[24] Chartier Y, Emmanuel J, Pieper U, Prüss A, Rushbrook P, Stringer R, Townend W, Wilburn S, Zghondi R. second ed. WHO); Safe Management of Wastes from Health-Care Activities. 2014. 